\title{
Opportunistic Monitoring of Pavements for Noise Labeling and Mitigation with Machine Learning
}

\author{
Wout Van Hauwermeiren ${ }^{\mathrm{a}, *}$, Karlo Filipan ${ }^{\mathrm{b}}$, Dick Botteldooren ${ }^{\mathrm{a}}$, Bert De Coensel ${ }^{\mathrm{a}, \mathrm{b}}$ \\ ${ }^{a}$ Ghent University, Tech Lane Ghent Science park 126, B-9052 Ghent, BELGIUM \\ ${ }^{b}$ ASAsense cvba, Spanjaardstraat 4, B-8000 Brugge, BELGIUM
}

\begin{abstract}
Currently, municipalities assess rolling noise on road surfaces using Close-Proximity measurements (CPX). To avoid these labor-intensive measurements, an opportunistic approach based on commodity sensors in a fleet of cars, is proposed. Blind sensor calibration eliminates the effect of measurement vehicle and varying observation conditions. Calibration relies on spatial coherence: modifiers and confounders do not interact strongly with location while the quantity of interest depends on location and less on measurement vehicle. Generalized additive speed models, car offset and de-noising autoencoders (DAE) were investigated. DAE achieves prominent results: (1) ratio of variability of measurements at a single location to the variability of measurements over all locations increases, (2) convergence of mean measurement at a location is faster, and (3) seasonal effects are eliminated. Finally, although the proposed method includes a diversity of tires, below $1600 \mathrm{~Hz}$ its results differ from CPX less than the difference between bi-annually repeated CPX measurements.

Keywords: road noise, rolling noise, blind sensor calibration, artificial neural networks, de-noising autoencoder
\end{abstract}

\section{Introduction}

Road traffic noise is caused by the engine (and auxiliaries) at the one hand and the noise of tires rolling over the pavement at the other. Both components have been the target of noise regulation and mitigation actions. Classically, noise mitigation through pavement choice and maintenance has been limited to high driving speeds as engine noise dominated the noise emitted by low speed traffic (e.g. inner city traffic). However, due to the rapid introduction of electric and hybrid vehicles, especially for light vehicles, the development of more quiet combustion engine, and the advent of low emission zones, the noise produced by tire-road interaction will become the next focus in traffic noise mitigation [1]. Both the tire and the road surface play their role in rolling noise mitigation. EU has introduced a tire label that informs the consumer about power consumption, safety, and noise emission [2]. Correspondingly, pavement type labeling with respect to its performance in rolling noise mitigation could also be envisaged. Moreover, as road surfaces (in particular the ones producing the lowest level of rolling noise) degrade over time [3], monitoring of the roads should also be a focus point. Finally, when considering local and inner-city

\footnotetext{
* Corresponding author

Email address: wout.vanhauwermeiren@ugent.be (Wout Van Hauwermeiren)
} 
roads, frequent local damages and repairs can be expected.

In the EU, Environmental Noise Directive 2002/49/EC requires that member states publish noise maps and noise mitigation action plans every 5 years for road noise, railway noise and aircraft noise. In this publication, we focus exclusively on road noise. To this end, a calculation methodology has been developed for strategic noise mapping, namely CNOSSOS (Common Noise Assessment Methods in Europe) 4]. CNOSSOS proposes standard values for rolling and engine noise of different types of road vehicles. To account for local variations in choice of pavement, pavement maintenance and tires used, measurements are needed. Road noise measurements are usually based on Close-Proximity Measurements (CPX) [5], Controlled Pass-By (CPB) or Statistical Pass-By (SPB) measurements. These standardised methods for characterisation of road surfaces have been extensively studied in the ROSANNE project [6]. Due to possible absorption of sound close to the tire and due to the directivity of emission, SPB are a preferred method for calibrating CNOSSOS to the local situation. CPX is a mobile method for measuring stretches of road. The ISO method requires two microphones which are placed at close proximity to a reference tire. Optionally, a third microphone can be added. Measurements are performed at a constant velocity (e.g. 50 $\mathrm{km} / \mathrm{h}, 80 \mathrm{~km} / \mathrm{h}, 100 \mathrm{~km} / \mathrm{h} \ldots$ [7]). The reference tire is usually placed in a trailer with either an enclosure (to shield the microphone from the wind) or exposed to the environment [8].

In 2016, the ROSANNE project has studied the compatibility of the CPX method with CNOSSOS 7]. For light vehicles, the correlations between CPX and SPB were good and within $\pm 1 d B 90 \%$ confidence under the following conditions: (1) CPX and SPB should be measured at the same reference speed, (2) outliers were removed in CPX data. CNOSSOS requires spectral data in octave bands from $63 \mathrm{~Hz}$ to $8 \mathrm{kHz}$. However, CPX is usually collected in $1 / 3$ octave band from $315 \mathrm{~Hz}$ to $5 \mathrm{kHz}$.

The CPX standard was revised in 2017: ISO 11819-2 (CPX method) [9] and 11819-3 (reference tires) [10]. The newest standard corrects for slight deviations in driving speed, air temperature during the measurements, and rubber hardness of the test tire. Bühlmann showed in 2019 [11 that after these corrections, repeated measures at the street granularity, taken at different days were well within one $\mathrm{dB}$ of each other and that the correlation between CPX and SPB is 0.87 for overall noise levels on roads with a range of $10 \mathrm{dBA}$ in CPX levels. However in the spectral domain, frequency bands lower than or equal to $630 \mathrm{~Hz}$ show a correlation with SPB well below 0.8 .

However there are also some critical voices regarding the CPX method. Due to the strict requirement of a reference tire, there are concerns that CPX does not accurately model the distribution of consumer tires on the road [12. Results show that a single kind of tire is not enough to measure the acoustic performance of road surfaces. In particular this is problematic for low-noise road surfaces. In practice, obtaining CPX measurements are prone to weather conditions, human errors (e.g. measuring the wrong section), calibration errors, CPX model manufacturer and the measurement instance (e.g. a 5 months interval between different measurements) 8 . Still, for a passenger tire $0.8 d B A$ maximum difference was observed between different CPX runs 8 .

CPX, CPB and SPB have as a drawback that they are quite labor intensive. An alternative to planned measurements is opportunistic sensing. Opportunistic sensing is closely related to citizen science. More precisely, large amounts of mobile sensors are deployed to measure an underlying effect. For instance: air pollution [13], 


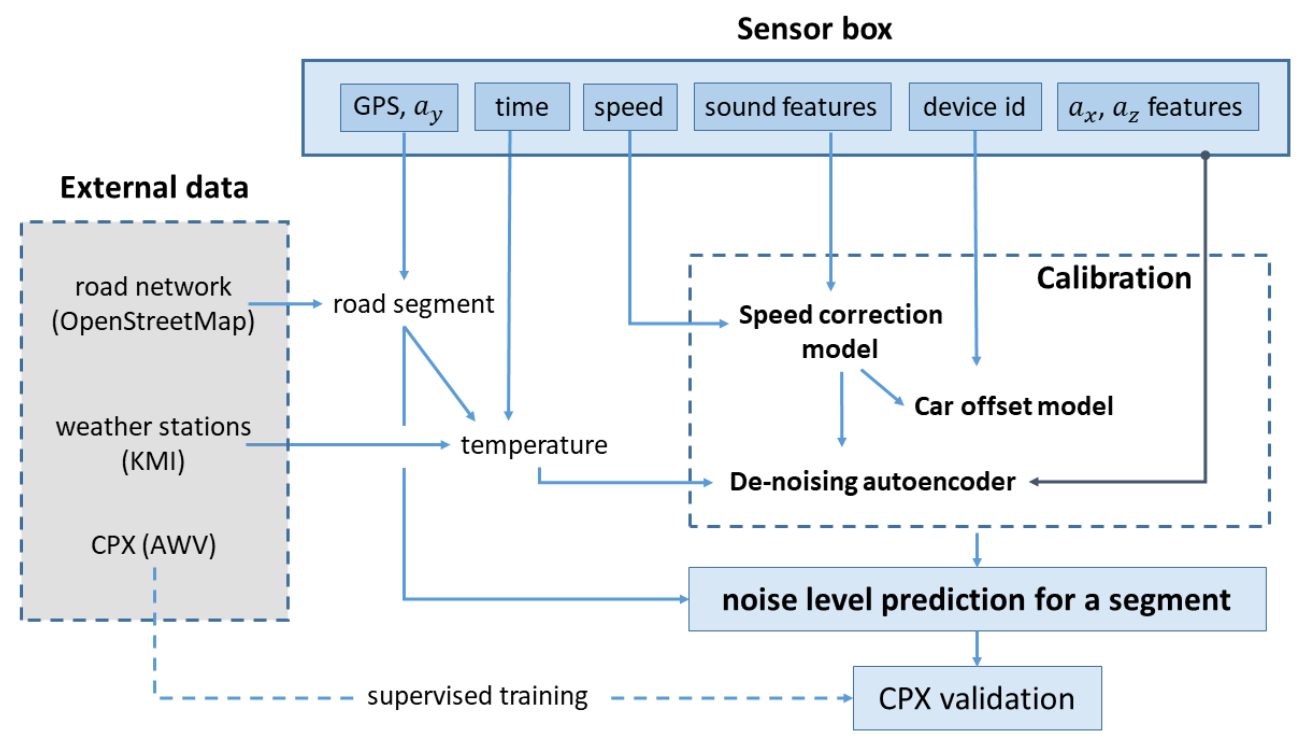

Figure 1: Flow chart presenting the methodology of collecting, calibrating and fusing the sensor data with external data sources.

noise pollution [14, road quality [15] [16]... A crowd of people perform the measurements as a side effect of their normal activities. Opportunistic sensing requires a high number of sensors to be placed in many vehicles and thus these sensors should be cheap and low maintenance. This unfortunately implies a lack of accuracy of each individual measurement. Moreover, measurement conditions are uncertain as they are dependent on the vehicle and moreover citizens may choose to change the measurement setup either on purpose or by accident. Thus, one typically relies on big data and machine learning for removing confounders and modifiers. Yet, in the case of rolling noise characterisation, the method has the added benefit over CPX and CPB that it averages over large sets of tires and driving conditions.

For this paper, several cars have been equipped with a sensor box in their trunk. The microphone was placed at a few $\mathrm{cm}$ distance to the inside of the rear wheel cavity, typically behind the interior lining. The project has been previously presented in 2019 at conferences, focusing on: noise labelling [17, road texture [18] and noise mapping [19]. In addition, a pilot study published in David et. al in 2019 [20], investigated a single car which drove multiple times at a selected trajectory. The noise - speed road interaction was eliminated using Support Vector Regression and a Generalized Additive Model. A clustering algorithm was then applied to the spectral features and has resulted in road section labels validated by on-site examination of the surfaces. The pilot study has thus shown the eligibility of the opportunistic sensing method.

This publication scales up the previous work to multiple vehicles, non-standard driving conditions and noisy car interiors and discusses different methodologies including a denoising autoencoder to meet the challenges of opportunistic sensing. To calibrate different cars, multiple passages of different vehicles on the same road segment are required. Due to the nature of opportunistic sensing, this dataset is typically unbalanced and sparse. Firstly, not all road segments are equally driven over by all the cars. Secondly, the individual passages record different 


\begin{tabular}{c|c|c}
\hline label & model & engine \\
\hline A & Kia Niro & hybrid gasoline \\
B & Volvo XC90 & gasoline \\
C & Fiat 500 & gasoline \\
D & Renault Megane & diesel \\
E & Audi Q5 & diesel \\
\hline
\end{tabular}

Table 1: Available devices

moments in time. Weather conditions, traffic and driving condition, engine state and interior noise could all vary between passages. Relying on averaging over many situations and cars would simply take too long: average passenger car, speed, no acceleration, temperature and dry roads. Therefore, a specially designed type of deepneural-network-based denoising auto-encoder is introduced to transform each measurement. The result is compared to CPX measurements on the right most lane of all highways and primary roads in Flanders. The produced spectral road noise correction factors reflect underlying road state: pavement type and wear. Figure 1 presents the flow of data in this paper. Parts of this publication have been presented at Inter-Noise 2020 [21].

\section{Material and methods}

The sensor boxes are equipped with noise, vibration and GPS sensors. They are placed in the trunk of a variety of passenger vehicles (see table 1): the cars have different tires, weights, suspensions, and car bodies. The following general conditions apply to all vehicles. The microphone is located close to the rear right or rear left wheel. If possible, the microphone is installed on the right or left side of the trunk below the fabric, near the spare tire. The sensor box setup may vary depending on the make of the car and the possibilities to protect the microphone and the box from tampering and accidental damage. In essence, the setup has been designed to be as non-intrusive as possible (no drilling).

The transfer functions between rolling noise production and measurement readings of different cars may therefore be quite different. This problem domain is closely related to blind sensor calibration [22], [23]. Suppose that the goal is to measure an underlying phenomenon using a set of $n_{c}$ non-calibrated sensors. The goal of the blind sensor calibration problem is to perform a self-supervised calibration procedure without ground truth data. In a second step, a simple model (e.g. linear) transforms the calibrated result into the physical phenomenon. This model should be agnostic of the sensor which has performed the measurements. For the problem at hand, there is already a significant amount of domain knowledge (e.g. a dependence on the driving speed) that could be taken into account prior to the blind sensor calibration. The problem is also simplified by assuming that the underlying phenomenon is discretely dependant on location. In particular, location $x$ can even be used as a factor (one-hot encoded vector). Finally, the blind sensor calibration procedure will converge better the more significant the overlap between the sensing areas of the different sensors. E.g. there are a lot of locations $x$ with multiple passages of 


\begin{tabular}{l|l|l}
\hline label & description & reason of choice \\
\hline$L_{E Q}(f)$ & equivalent spectral sound level & overall road noisiness \\
$L_{05}(f), L_{50}(f), L_{95}(f)$ & percentiles of $L(f, t)$ & changes in road surface \\
$L_{\max }(f)$ & maximum of $L(f, t)$ & sudden changes (e.g. potholes, bridge joints) \\
$a_{z}(f), a_{z, \min }, a_{z, \max }, \mu\left(a_{z}\right)$ & spectral and statistical vertical acceleration & vibrations due to road surface \\
$a_{y, \text { min }}, a_{y, \max }, \mu\left(a_{y}\right)$ & sideways acceleration & lane changes \\
$a_{x, \text { min }}, a_{x, \max }, \mu\left(a_{x}\right)$ & horizontal acceleration & driving conditions \\
$v$ & speed & driving conditions \\
$T$ & temperature & external condition \\
$x$ & location from GPS & continuous localization \\
$x_{m}$ & $20 \mathrm{~m}$ road segment label & discrete localization \\
\hline
\end{tabular}

Table 2: List of features extracted from the sensor box. These are evaluated in a period of 1 second. $\mu$ is the average over 1s of samples.

different devices.

The problem of blind sensor calibration has previously been approached using statistical sensor calibration 24 22 23. This domain uses statistical algorithms such as Least Squares, Bayesian estimation, Markov Chain Monte Carlo and Kalman filters to calibrate the sensors. Moreover, deep learning is connected to statistical sensor calibration in 25. This publication introduces the Projection Recovery Network (PrNet), for the task of sensor drift calibration. This is a deep neural network architecture with convolutional layers. It allows to account for drift even in the presence of a time dependent target measurement.

\subsection{Formal description of the data collection}

Noise captured in the trunk of car $c$ at a moment $t$ (also indicative of the trip or passage) can be described for each frequency band $f$ by:

$$
p^{2}(f, t, x(t), c)=H(f, c, v(t)) * p_{s}^{2}(f, x(t))+p_{e}^{2}(f, c, v(t))+p_{n}^{2}(f, t)
$$

where $p_{s}^{2}$ indicates the sound pressure level at close proximity to the tire at a reference speed, $p_{e}^{2}$ points to engine noise and $p_{n}^{2}$ to other vehicle interior noise. $H$ is an unknown transfer function between the location close to the tire (at a reference speed) and the actual measurement position at the actual speed. The variable $v$ includes all contextual variables: speed, acceleration, temperature,... Weak dependencies have been omitted: e.g. aging of road surface and hardening or tires in $p_{s}^{2}$ or effects of cold start in $p_{e}^{2}$. The pressures $p$ are RMS values and the different contributions are incoherent, hence the addition of pressures squared. For brevity the following notations are introduced: $L_{s}=10 \log p_{s}^{2}, L_{e}=10 \log p_{e}^{2}, L_{n}=10 \log p_{n}^{2}$. 
The sensor box records location and other parameters from the GPS receiver, tri-axial acceleration signal from the accelerometer and sound from the microphone. Then, a filter bank extracts $1 / 3$ octave band levels at a sampling interval of $0.02 s, L(f, t)=10 \log p^{2}(f, t)$. Afterwards, a number of features are extracted from the filter bank and other signals (see table 2). These features are calculated over 1 second intervals. The sampling interval and feature periodicity are selected as a compromise between transmission bandwidth and spatial accuracy (which is targeted at 20m). In addition, they are aligned, therefore they represent a feature vector, for usage in machine learning algorithms. Map matching and lane detection [26, is used for mapping the geographic localization, $x$ on a $20 \mathrm{~m}$ road segment, $x_{m}$ (including the driving lane). OpenStreetMap (OSM) is used as a road network map. Observations are only kept between $20 \mathrm{~km} / \mathrm{h} \approx 5.6 \mathrm{~m} / \mathrm{s}$ and $130 \mathrm{~km} / \mathrm{h} \approx 36 \mathrm{~m} / \mathrm{s}$ driving speed. Considering these facts, $20 \mathrm{~m}$ segments seems an appropriate choice. This means that in 1 second approximately between $1 / 4$ of a segment and 2 segments are sampled. To this end, measurements are duplicated and assigned to all segments which are passed by during $1 s$, to avoid skipping the locations at high speeds.

It has been hypothesised that there could be some sensor drift and or effects due to the tire heating up on the noise levels. Therefore, outside air temperature is also included in the feature set. For this, the timestamp and GPS location is used to locate the closest weather station. These stations are maintained by the KMI weather agency in Belgium.

For this paper, a dataset of 5 cars is constructed. Two cars are diesel cars, two cars are gasoline and one car is a hybrid electric-gasoline car. For one gasoline car there has been a switch between winter and summer tires.

In the following sections several calibration techniques are described.

\subsection{Speed model}

It is well-known that rolling noise strongly depends on speed [4] 27], yet during opportunistic sensing, the speed may vary over a considerable range. Hence, the raw measurements will be corrected for speed and samples with a strongly deviant speed will be discarded.

\subsubsection{Average road speed model}

A speed model can be built by assuming there is only a speed dependency (and not road surface dependency). This has been previously applied in David et al. 20] and the follow up research presented at Inter-Noise 2019 [17. To begin (in [20]), a single car drove repeatedly on a reference trajectory during a period of 4 months. A speed curve has been fit using Support Vector Regression (SVR) and a Generalized Additive Model (GAM). In [17, the dataset has been expanded with multiple devices.

Suppose that by locating the microphone at a suitable position, the car interior noise $p_{n}^{2}$ and the engine noise $p_{e}^{2}$ are smaller than the signal originating from the tire. Then, a logarithmic function can be applied to both sides of equation 1 while setting $p_{n}^{2}=p_{e}^{2}=0$ resulting in:

$$
10 \log \left(p^{2}(f, t, x(t), c)\right) \approx 10 \log (H(f, c, v(t)))+10 \log \left(p_{s}^{2}(f, x(t))\right)
$$


$\log (H(f, c, v(t)))$ contains the known dependencies $v$ (speed only) and becomes an additive term. Now, a generalized additive model (GAM) $g(f, c, v)$ is fitted on $L=10 * \log \left(p^{2}\right)$ for each frequency band, $f$, and each sensing system, $c$. As the GAM fit is independent of $x$, it will result in an estimate of $H+\mu(L)$, where $\mu$ refers to the average.

Finally, by subtracting the fitted curve, the main speed dependence is removed.

$$
d L_{g}(f, x(t), c,[t])=L(f, t, x(t), c)-g(f, c, v(t))
$$

$d L_{g}$ is assumed to encode how much the rolling noise emission at the current road segment $x_{m}$ differs from that of the average road where cars drive at the same speed. The explicit time/trip dependence is largely removed, yet any remaining dependence is expected to be removed by averaging over multiple passages. The average $\mu\left(p_{s}^{2}\right)$ is however dependant on locations which are sampled by each measuring device $c$. The GAM has been fitted on the first hours of driving of a car while the speed range has been subdivided into speed bins for which a minimum driving time of 30 minutes is required. Although this assures that the GAM does not over-fit on specific driving speeds that are typically related to types of roads, the assumption that all cars, $c$, drive a representative sample of roads remains critical in this approach.

\subsection{Car offset model}

The speed model implicitly includes a frequency dependent offset for each car. It is calibrated by assuming all cars statistically speaking drive on the same roads. But, as this hypothesis does not hold in many cases, the car offset model determines a frequency-dependent calibration constant that should be added to each sensor value. Doing so, would obtain the reference sound pressure level $L_{s}\left(f, x_{m}(t)\right)$ :

$$
d L_{g_{1,2}}\left(f, t, x_{m}, c\right)+\lambda_{1}(c, f) \approx L_{s}\left(f, x_{m}(t)\right)
$$

This offset is added to the result of the speed correction of the previous section. Since the reference sound pressure level is unknown, instead we will be transforming measurements made by one sensor system to another using a constant. This constant will be determined by comparing measurements $d L_{g}\left(f, t, x_{m}, c\right)$ from different cars on the same road segments, $x_{m}$.

Since there are $n_{c}$ sensors, a multivariate Linear least squares model with 12 regularization is proposed [28]. By taking the difference between target sensor and input sensor, $L_{s}\left(f, x_{m}(t)\right)$ is dropped.

$$
d L_{g}\left(f, t, x_{m}, c_{2}\right)-d L_{g}\left(f, t, x_{m}, c_{1}\right)=\Lambda_{2} * C_{2}-\Lambda_{1} * C_{1}
$$

The fitted constants must be small so it is necessary to use regularization. The goal is to transform features from one input sensor to another target sensor. The sensors are taken as a binary vector variables $\left(C_{1} C_{2}\right)$ with $C_{1}$ the input sensor and $C_{2}$ the target sensor, e.g. for 4 sensors, $C_{1}=(1000), C_{2}=(0100)$. This is the representation to transform observations from sensor 1 to sensor 2. $\Lambda_{1}$ is the vector with coefficients $\lambda_{1}\left(c_{1}, f\right)$ transforming an input sensor to a value close to $L_{s}\left(f, x_{m}(t)\right) . \Lambda_{2}(f)$ is the vector with coefficients $\lambda_{2}\left(c_{2}, f\right)$ transforming $L_{s}\left(f, x_{m}(t)\right)$ to observations of a certain target sensor $c_{2}$. 
To make predictions with this model, $C_{2}$ can be set to a leader sensor or $C_{2}=\left(1 / n_{c}, 1 / n_{c}, \ldots 1 / n_{c}\right.$ ) (average over all sensors). $d L_{C O}$ is obtained as output value:

$$
d L_{C O}\left(f, t, x_{m}, c_{1}, c_{2}\right)=d L_{g_{j}}\left(f, t, x_{m}, c_{1}\right)-\Lambda_{1} * C_{1}+\Lambda_{2} * C_{2}
$$

\subsection{Confounder removal using a de-noising auto encoder}

In the previous sections it has been assumed that (1) the additive engine noise $p_{e}^{2}(f, c, v(t))$ and car interior noise $p_{n}^{2}(f, t)$ can be neglected for all features, (2) there is no interaction between the known dependencies, $v$ and (3) all devices sample on average the same distribution of $p_{s}^{2}$. We postulate that these conditions can be relaxed by using a de-noising auto-encoder (DAE) for cleaning the data. Usually, DAE's rely on a clean dataset without noise. This dataset is augmented by adding varying sources of noise, e.g. white noise in the case of a speech recognition problem [5]. The DAE then learns how to extract the clean signal given a noisy signal. Unfortunately, there is no clean data available for training and these basic techniques cannot be applied here. Yet, $p_{e}^{2}$ and $p_{n}^{2}$ usually do not depend very strongly on the location $x_{m}(t)$ but vary considerably with $t, v$, and $c$. Therefore, statistically speaking some measurements with less noise will be available in the data set at most locations. Moreover, correlation between features will help the model to estimate those features that are more sensitive to noise, e.g. frequency bands where engine noise is high.

In this manuscript, the DAE is trained by pairing up observations made at different time instances (and optionally by a different device) at the same location. Every passage samples the same spatial information, but at different conditions (e.g. in warm weather, accelerating, decelerating, at high speed, at low speed...). An observation feature vector, made at time $t$, is split up into different parts: the input feature $I F(t)$, context conditions $C C(t)$, the device information $c(t)$ and the output feature $O F(t)$. Similar to the car offset model $c(t)$ is again a one-hot encoded vector. The DAE learns a transformation from an observation, $I F_{1}$, of car $c_{1}$ under context conditions $C C_{1}$ to the output feature vector $\left(O F_{2}\right)$ of car $c_{2}$ under context conditions $C C_{2}$. The method can be chained with the output of any of the previously discussed calibration methods. If the DAE is chained with the speed correction model than the deviations from average $d L_{g}$ will constitute the input vector $I F$. Frequencies outside the range of interest for rolling noise, statistical levels and vibration features are also part of $I F$.

$$
d L_{D A E^{\prime}}\left(I F_{1}, C C_{1}, c_{1}, C C_{2}, c_{2}\right)=d L\left(O F_{2}\right) \approx d L_{g}\left(v_{2}, c_{2}, f\right)
$$

where $d L$ has been introduced to represent extracting the relevant noise levels from the complete feature vector. At prediction time, the samples are averaged over all cars $c_{2}$ at reference conditions $C C_{r e f}$.

$$
d L_{D A E}\left(t, f, C C_{r e f}\right)=\frac{1}{N_{c}} \sum_{c_{2}=1}^{N_{c}} d L_{D A E^{\prime}}\left(I F(t), C C(t), c(t), C C_{r e f}, c_{2}\right)
$$

In the ideal case, it should not matter which device has made the measurements. Every DAE calibrated measurement $d L_{D A E}$ is an average over all the cars and thus all the tires in the data set. Section 3 explains the used configuration of the neural network and the selection procedure of the neural network hyper parameters. 


\subsection{Calibration metrics}

A cleaning method must decrease the variability of multiple measurements at the same location, $x_{m}$. In addition, the variability over multiple locations must remain as large as possible. In other words: capture as much information as possible about the locations. Hence, several metrics are proposed. In the following formulas $M$ is either $L, d L_{g}, d L_{C O}$ or $d L_{D A E}$.

Average of within segment variability:

$$
w(M)=\mu\left(\left\{\sigma\left(\left\{M\left(c, x_{m}, t\right) \mid \forall(c, t) \in x_{m}\right\}\right) \mid \forall x_{m}\right\}\right)
$$

Variability over all segment estimated means:

$$
o(M)=\sigma\left(\left\{\mu\left(\left\{M\left(c, x_{m}, t\right) \mid \forall(c, t) \in x_{m}\right\}\right) \mid \forall x_{m}\right\}\right)
$$

Ratio:

$$
r(M)=o(M) / w(M)
$$

$\mu$ and $\sigma$ indicating respectively the average and the standard deviation of a set of values. $(c, t) \in x_{m}$ indicate measurements made by device $c$ at moment $t$ at a segment $x_{m}$. In addition, the metrics can be computed for the observations of a single device, $c$.

\subsection{Noise level estimates}

The calibration methods output relative spectral noise levels to 'an average road'. A reference noise level spectrum $L_{R E F}(f)$ can be obtained by taking the average of a set of measurements (such as CPX, CPB or SPB). The noise level estimate $\tilde{L}\left(f, x_{m}\right)$ is then given by:

$$
\tilde{L}\left(f, x_{m}\right)=d L\left(f, x_{m}\right)+L_{R E F}(f)
$$

$d L$ is either $d L_{g}, d L_{C O}$ or $d L_{D A E}$.

To obtain a noise label, the $1 / 3$ octave bands are summed in $p^{2}$ :

$$
\tilde{L}\left(f, x_{m}\right)=10 \log \sum_{f} 10^{1 / 10 * \tilde{L}\left(f, x_{m}\right)}
$$

\section{Hyper parameter tuning and training of the DAE}

This section elaborates on the choice of de-noising auto encoder (DAE) architecture and its hyper parameter tuning. A DAE is a kind of neural network arranged in an encoder-decoder structure [30. Figure 2 introduces the structure of the auto-encoder neural network. The layout is chosen to contain 6 hidden layers and 1 bottleneck layer.

The input layer contains the input features $I F$, the context variables $C C$, and the car identifier, $c$. As mentioned, IF contains the additional noise and vibration features of Table 2 to provide more context about the road state. The 


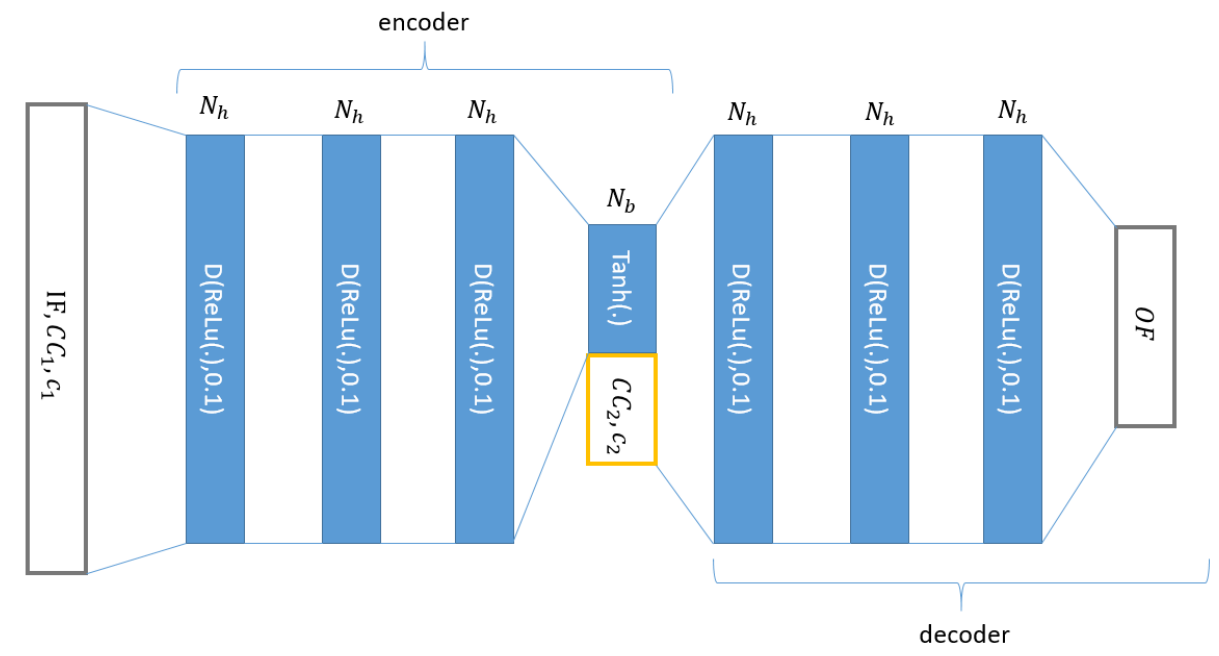

Figure 2: Structure of the neural network. $N_{h}, N_{b}$ : amount of hidden nodes per layer and amount of bottleneck nodes respectively. D refers to a dropout operation during training, ReLu to Rectified Linear unit [29, tanh to tangens hyperbolicus.

bottleneck layer is chosen small, in order to force an internal representation of the input layer in a low-dimensional space. $c_{2}$ and $C C_{2}$ are concatenated with the bottleneck layer. The idea is that the decoder part reconstructs observations to $c_{2}$ using a car-agnostic and contextual variable-agnostic representation in the bottleneck layer. In deep learning, there is no one solution which fits all problems. In this work, a Rectified Linear Unit (ReLu), defined as $\max (0, x)$, is chosen as the activation function. ReLu is computationally efficient, converges faster $\operatorname{than} \tanh (x)$ and handles the vanishing gradient problem for deep neural networks [29]. For the bottleneck layer, a $\tanh (x)$ activation function is used. This forces the internal representation to the interval $[-1,1]$. The dropout operation is a way to regularize the neural network [31. For a given probability, the nodes are set to 0 which ensures a certain amount of redundancy in the neural network.

In addition, the optimization algorithm needs to be chosen. For this paper the Adam optimizer [32] is used. A loss function has been chosen: the mean squared error of the reconstruction of the target samples. The data has been split into a train and test set. In case the representational strength of the neural network is high enough, the neural network could remember all the input and output pairs [33. Therefore, the test set is used to assess the generalization capability of the neural network. The training procedure is listed in the appendix in code listing A.1.

\subsection{Hyper parameter tuning using cross validation}

Designing and training deep neural networks is a challenging task [34. There are quite a few hyper parameters which need to be tuned: amount of bottleneck nodes, amount of hidden layers, amount of hidden nodes per layer, learning rate and dropout probability, In this section, a closer look is taken into hyper parameter tuning with cross validation. For several configurations of the DAE network, cross validation has been applied. The following procedure is followed:

1. keep $20 \mathrm{~m}$-segments, $x_{m}$ with at least 10 passages by at least 2 devices 


\begin{tabular}{l|lll}
\hline (1) configuration & \multicolumn{2}{l}{} \\
\hline bottleneck nodes & $10, \mathbf{2 0}, 50,100$ & \\
hidden nodes per layer & $50,100,150, \mathbf{3 0 0}$ & \\
learning rate & $10^{-5}$ & & \\
dropout & $\mathbf{0 . 1}$ & & \\
\hline (2) features & & context variables & \\
input features & & speed & yes, no \\
\hline$L_{E Q}(f)$ & yes, no & $a_{x, \min }, a_{x, \max }, \mu\left(a_{x}\right)$ & yes, no \\
$L_{05}(f), L_{50}(f), L_{95}(f), L_{\max }(f)$ & yes, no & temperature $(T)$ & yes, no \\
$a_{z}(f), a_{z, \min }, a_{z, \max }, \mu\left(a_{z}\right)$, & yes, no & & \\
$a_{y, \min }, a_{y, \max }, \mu\left(a_{y}\right)$ & & & \\
\hline target features & $L_{E Q}(f)$ & &
\end{tabular}

Table 3: Possible choices for model configurations. First, parameters have been cross validated in heading "(1) configuration", then another crossvalidation run is performed with parameters in "(2) features" Grid search has been applied: choose either "yes" to include or "no" to not include for every feature set, or alternatively one of the options listed next to the parameter name. The final configuration is given in bold.

2. label $80 \%$ of segments as train set and $20 \%$ as test set

3. subdivide the train set into 5 folds

4. Repeat for each of the 5 folds: take one fold as test set and train using the other 4 folds (see listing A.1 for the training procedure)

5. collect performance metrics over the folds

The entire train set contains 64839 road segments of $20 \mathrm{~m}$ (around $1296 \mathrm{~km}$ of roads). In total there are 2219 271 passages, which amounts to 34.2 passages per segment. The test set contains 16393 segments, a total of 557109 passages, which amounts to 34 passages per segment.

First of, different layer sizes were cross-validated. The configurations which are used are listed in table 3 , under the header "(1) configuration". For this experiment, all the features are chosen which are listed in the mentioned table. The results for the 5 best configurations are given in table 4 . The average MSE lie closely to each other. A final configuration of 20 bottleneck nodes and 300 hidden nodes is chosen.

Secondly, another experiment to test the validation error for different feature combinations (table 3 ) has been run. The results for the top 5 models are indicated in table 5. The test loss varies quite a bit run by run. The model which uses all features is included in the top 5 and has the lowest variability between folds, therefore it has been decided to keep on using all the features. For two out of five models, $L_{E Q}$ is not used. However, $L_{50}$ is included in the feature set. $L_{50}$ and $L_{E Q}$ could contain similar information. Temperature seems to be an important parameter as well: in the top 16 models temperature is always included.

Finally the DAE model has been trained with the configuration listed in table 3 . First, the best performing 


\begin{tabular}{lrrrr}
\hline & bottleneck nodes & hidden nodes per layer & $\mu\left(M S E_{\text {val }}\right)$ & $\sigma\left(M S E_{\text {val }}\right)$ \\
\hline$\# 1$ & 20 & 300 & 0.487245 & 0.048229 \\
$\# 2$ & 10 & 150 & 0.493469 & 0.067631 \\
$\# 3$ & 10 & 300 & 0.494887 & 0.035496 \\
$\# 4$ & 100 & 150 & 0.496557 & 0.057467 \\
$\# 5$ & 50 & 300 & 0.499714 & 0.055597 \\
\hline
\end{tabular}

Table 4: Top results for layer size cross validation; $\mu$ refers to the average over all folds and $\sigma$ to its standard deviation

\begin{tabular}{c|c|c|c|c|c|c|c|c}
\hline & $L_{E Q}$ & $L_{05}, L_{50}, L_{95}, L_{\max }$ & $a_{z} \& a_{y}$ & speed & $a_{x}$ & $T$ & $\mu(M S E)$ & $\sigma(M S E)$ \\
\hline$\# 1$ & $\mathrm{o}$ & $\mathrm{o}$ & & $\mathrm{o}$ & $\mathrm{o}$ & $\mathrm{o}$ & 0.370698 & 0.077868 \\
$\# 2$ & $\mathrm{o}$ & $\mathrm{o}$ & $\mathrm{o}$ & & $\mathrm{o}$ & 0.388052 & 0.057547 \\
$\# 3$ & $\mathrm{o}$ & $\mathrm{o}$ & $\mathrm{o}$ & & $\mathrm{o}$ & 0.400418 & 0.092777 \\
$\# 4$ & & $\mathrm{o}$ & & $\mathrm{o}$ & $\mathrm{o}$ & $\mathrm{o}$ & 0.402925 & 0.080619 \\
$\# 5$ & $\mathrm{o}$ & $\mathrm{o}$ & $\mathrm{o}$ & $\mathrm{o}$ & $\mathrm{o}$ & $\mathrm{o}$ & 0.407839 & 0.043983 \\
\hline
\end{tabular}

Table 5: Cross-validation on feature combinations: which features produces the most accurate predictions and the most consistent results? Select features groups of the top 5 models are shown. In the last two columns the average $(\mu)$ and standard deviation $(\sigma)$ of mean squared error (MSE) over 5 folds are given.

model for a certain fold is selected and then DAE training has been continued by feeding in all the training segments 30 times.

\section{Results and discussion}

As previously stated, the data has been collected by sensor boxes placed in 5 cars listed in table 1. Each driver of a car performs a different driving pattern. For instance, devices $\mathrm{A}$ and $\mathrm{C}$ are mostly travelling to work and back, therefore they cover the same subset of locations over a long amount of time. On the other hand, device D belongs to a travelling salesperson. This one covers a lot of new roads in a short period of time. Indeed, if the measurement cars are chosen appropriately, then the road network could be covered more efficiently: for instance using rental cars, small delivery vehicles or cars of sales people.

In the next sections the performance of the blind sensor calibration methods are compared with each other on different criteria. As a baseline, non-corrected levels $L$ are plotted and the effect of removing known dependencies is shown by comparing it to speed corrected $d L_{g}$ levels, measurements corrected with car offset $d L_{C O}$ and DAE corrected $d L_{D A E}$ measurements. 


\subsection{Statistical metrics on locations}

The metrics, introduced in section 2.5, are calculated for the different calibration methods. Results are given in figure 3. All the calibration methods reduce $w(M)$, the within-segment variability. In particular, the DAE

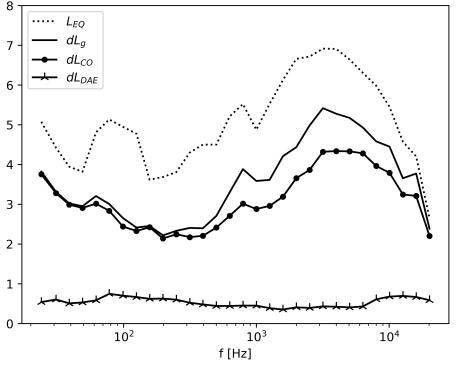

(a) $w(M)$

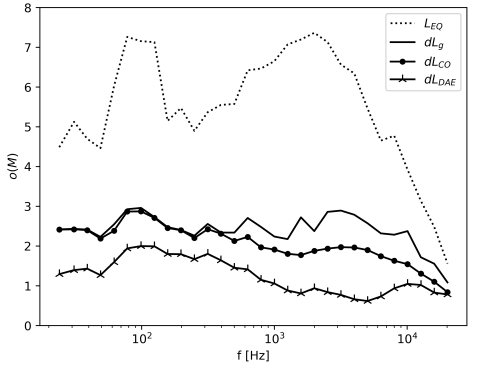

(b) $o(M)$

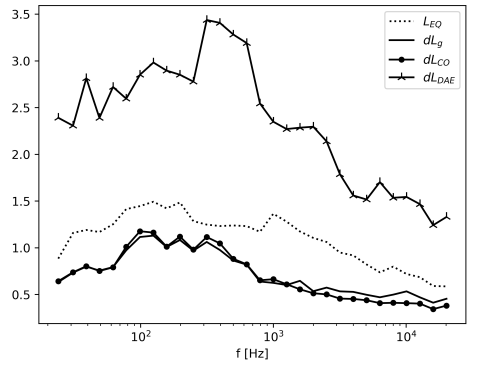

(c) $r(M)$

Figure 3: Comparison of different fully trained models of the within segment metric $w(M)$, over segments metric $o(M)$ and ratio $r(M)$. The feature used is $L_{E Q}$.

\subsection{Convergence over time}

The proposed opportunistic sensing method relies on averaging rolling noise over a large number of vehicles.

For the approach to be efficient, it should converge to its final value with as few passages as possible. Convergence of a measurement series could amongst other be checked by evaluating the decrease in standard error on the mean with the number of tests. For two locations with a lot of passages, the effects of different calibration methods on convergence is compared. This is shown in figure B.1 and figure B.2. Three 1/3 octave bands are chosen: $40 \mathrm{~Hz}$ (strongly influenced by engine noise), $800 \mathrm{~Hz}$ (related to degradation of dense asphalt) and $2 \mathrm{kHz}$ (related to air pumping effect of tires). As evident for the $800 \mathrm{~Hz}$ and $2 \mathrm{kHz}$ bands, the speed correction solves some of the 
convergence issues (seen in both locations). For $40 \mathrm{~Hz}$ however, there is only a slight improvement in standard error at location $\mathrm{B}$ for $d L_{g}$ and $d L_{C O}$ compared to $L_{E Q}$.

All of the five measurement vehicles are included at both locations. However, they are introduced at different time instances and their proportion of measurements is not equally balanced. Zooming in on location A (figure B.1): first car A and car E play a prominent role, then after 10 passages car A and car C start to dominate the measurements. For $40 \mathrm{~Hz}$ band there is a downwards trend for the non-DAE calibrations. This could be due to a more silent engine model for car C (gasoline) and car A (hybrid gasoline). At $800 \mathrm{~Hz}$ and even more at $2 \mathrm{kHz}$, a big drop is visible for $d L_{g}$ and $d L_{C O}$. This coincides with car $\mathrm{C}$ dominating the measurements more and more at 10 passages. The standard error of $d L_{D A E}$ converges faster to 0 . This is evident in the mean $d L_{D A E}$ values for all frequency bands: the DAE samples remain stable. For location B (figure B.2), car B is initially the most prominent car. At around 10 passages, car A is appears and starts to take on a large proportion of the observations. At the $800 \mathrm{~Hz}$ band, $d L_{g}$ and $d L_{C O}$ vary slightly in response to the change in measurement devices. At $2 \mathrm{kHz}$ the car offset model makes the convergence worse. This could be attributed to the fact that noise levels between cars are not simply a constant. To conclude, the DAE-corrected samples reflect the entire vehicle set and are by consequence less sensitive to which device has made the measurements.

To show the effect of faster convergence on trend analysis, in figure 4 , the overall noise label has been computed for 6 locations with car offset corrected measurements $d L_{C O}$ and DAE-corrected measurements $d L_{D A E}$. The locations are given in table 6. These locations have a lot of measurements over the course of 16 months and represent a range of highways, primary and secondary roads. This allows to consider 30-day running averages. The main observation when comparing $d L_{C O}$ to $d L_{D A E}$ is that the range of data is compressed from about 15 $\mathrm{dB}$ to about $5 \mathrm{~dB}$. But most of all, due to faster convergence, most measurements noise - identified as variations in noise labels from day to day - is reduced to within $1 \mathrm{~dB}$. The jumps in measured value are explainable. Road construction works have occurred at locations E17 and N60. A drop in noise level is visible at both locations for both methods. The DAE noise labels of the other locations increase steadily over the course of one year. The car offset model shows drops in noise levels as well at N43, however no road construction happened during the selected period. DAE noise levels show outliers as well, however this is caused by averaging only a couple of observations: visible mid 2019 for R4 and mid 2019 for E17.

\subsection{Seasonal variations}

Seasonal variations have been observed for $L_{E Q}, d L_{g}$ and $d L_{C O}$, in particular for car A and car C which have driven during more than a year. This is not unexpected as it is well known that rolling noise depends on tire and road temperature. In this section we explore whether the DAE learns to remove this seasonal dependence from the measurements. To this end, figure B.3 shows monthly noise level averages for $L_{E Q}, d L_{g}$ and $d L_{D A E}$ at $800 \mathrm{~Hz}$ and $2000 \mathrm{~Hz}$ for the two cars for a selection of locations. $d L_{C O}$ is not shown, because we are looking at observations of a single car at a time. For the hybrid car A, there is a recurring seasonal pattern with higher values of $L_{E Q}$ and $d L_{g}$ during winter and lower values during summer months. Gasoline car C shows a similar pattern, with a 


\begin{tabular}{|c|c|c|c|}
\hline road & order & speed limits & location \\
\hline E40 & highway & $120 \mathrm{~km} / \mathrm{h}$ & $51^{\circ} 0^{\prime} 56.8548 " \mathrm{~N}, 3^{\circ} 42^{\prime} 19.1622^{\prime \prime} \mathrm{E}$ \\
\hline N60 & secondary & $90 \mathrm{~km} / \mathrm{h}$ & $51^{\circ} 0^{\prime} 5.331 " \mathrm{~N}, 3^{\circ} 41^{\prime} 30.0474 " \mathrm{E}$ \\
\hline N43 & secondary & $70 \mathrm{~km} / \mathrm{h}$ & $51^{\circ} 1^{\prime} 11.892^{\prime \prime} \mathrm{N}, 3^{\circ} 40^{\prime} 56.3484$ " E \\
\hline E17 & highway & $120 \mathrm{~km} / \mathrm{h}$ & $51^{\circ} 2^{\prime} 46.5534^{\prime \prime} \mathrm{N}, 3^{\circ} 48^{\prime} 6.2172$ " E \\
\hline R4 & primary & $90 \mathrm{~km} / \mathrm{h}$ & $51^{\circ} 0^{\prime} 40.5252^{\prime \prime} \mathrm{N}, 3^{\circ} 44^{\prime} 28.1256^{\prime \prime} \mathrm{E}$ \\
\hline B401 & highway & $120 \mathrm{~km} / \mathrm{h}$ & $51^{\circ} 1^{\prime} 12.0894 " \mathrm{~N}, 3^{\circ} 43^{\prime} 55.0986 " \mathrm{E}$ \\
\hline
\end{tabular}

Table 6: Selected locations

drop in $L_{E Q}$ and $d L_{g}$ in summer. However, car C, has not yet collected the same amount of data as car A. The seasonal variation seems completely removed from $d L_{D A E}$. Also note the smaller variation in measurements (grey zone) as stronger corrections are applied.

In section 3.1, we have noticed that the temperature is chosen often in feature selection for the DAE. We

could conclude that by adding temperature as a variable to the DAE model, seasonal variations are eliminated. In particular, the DAE transforms observations into virtual observations at a reference temperature.

For CPX, -0.1dBA / degree air temperature change is expected [35, but the relationship between tire temperature, road temperature, and air temperature may add additional complexity [36]. In addition tire hardness and temperature effect may need to be considered together [36. Also the temperature correction for rolling noise estimation in CNOSSOS is of the order of $-0.08 \mathrm{~dB} /$ degree. Hence the observed $4 d B$ to $8 d B$ seasonal variation is higher than what would be expected for the Belgian climate. Alonso et al., 2014 [37] have found a prominent increase in normalized $1 / 3$ octave band measurements in the $500 \mathrm{~Hz}-8 \mathrm{kHz}$ bands for a wet road surface compared to a dry road surface. The effect becomes more prominent for higher frequency bands. In particular, the observed seasonal effect is stronger in the $2 \mathrm{kHz}$ band (see figure B.3). Precipitation and surface drying can be expected to be inversely correlated with temperature in the Belgian climate. Finally, the measurement equipment could also be prone to temperature influence. All this considered, this could explain why the DAE is still successful in removing these observed seasonal variations based on temperature alone.

\subsection{External validation: $C P X$}

The proposed opportunistic method for assigning a label to the rolling noise characteristics of a pavement differs from CPX as it involves multiple tires and as measurements are made at typical driving speeds for the road considered. Yet comparing both methods may shed some light on the influence of these different approaches. To this end, a dataset has been made available by Agentschap Wegen \& Verkeer (AWV), the Flanders government agency for road infrastructure. The data consists of CPX measurements made once for each primary road in 2019 at different time instances. Measurements are made in $1 / 3$ octave bands for a frequency range of $315 \mathrm{~Hz}$ to $5000 \mathrm{~Hz}$. In this section, the opportunistic gathered data has been fitted to the CPX data of 2019 using a linear regression model. $1190 \mathrm{~km}$ of roads has been provided, for which there is a $80 \%$ coverage with the opportunistic data. The 


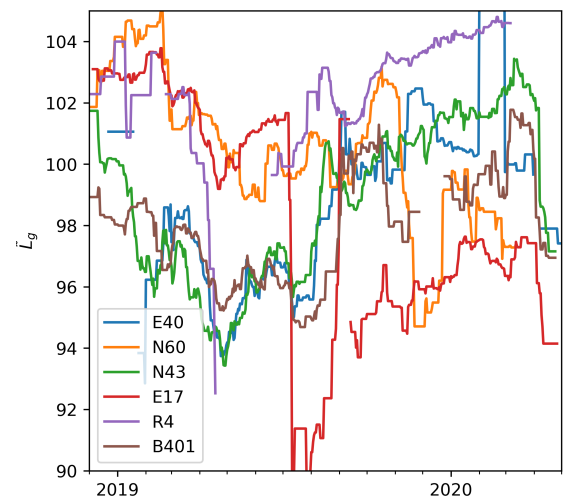

(a) speed correction $\left(d L_{g}\right)$

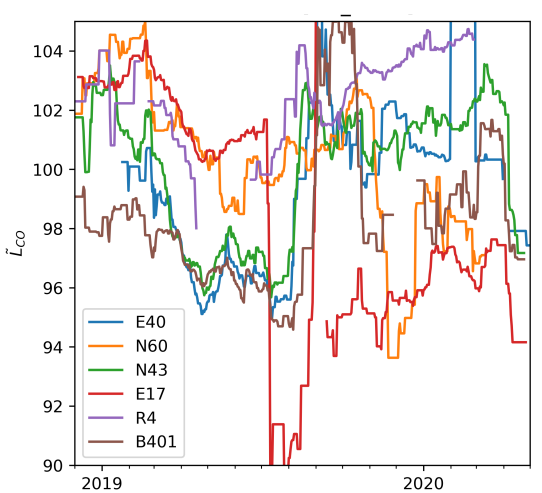

(b) Car offset $\left(d L_{C O}\right)$

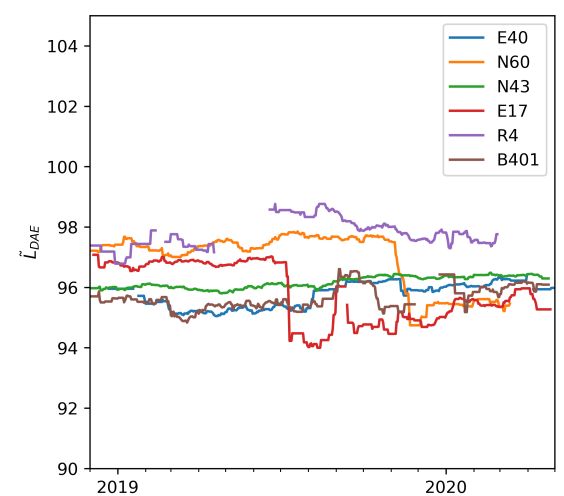

(c) $\mathrm{DAE}\left(d L_{D A E}\right)$

Figure 4: Evolution of the measured noise level during 16 months: car offset (left), DAE (right). A moving average computes the noise level for a day of the year, with observations taken one month before that day. Locations are listed in table6

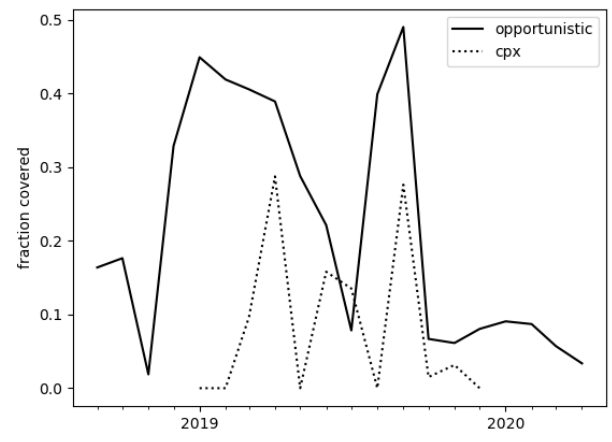

Figure 5: Fraction of CPX locations covered during each month by either the CPX vehicle or opportunistic measurements.

following hypothesis is tested: does the opportunistic data, corrected with the calibration methods, resemble CPX? Several side notes has to taken into account. (1) CPX data has been recorded at the right-most lane. Highway exits, mergers, highway entrances may introduces additional lanes to the left or to the right. Therefore, the definition of the right-most lane for the CPX and for the lane detection algorithm [26] used for map matching could occasionally be different. (2) Some pavements might have been resurfaced between both measurements, as different time periods are covered by the opportunistic data gathering and the CPX measurements (see figure 5).

A linear regression model has been constructed to predict the CPX spectrum $L_{C P X}\left(x_{m}, f\right)$ for $315 H z \leq f \leq$ $5000 \mathrm{~Hz}$ in $1 / 3$ octave bands. The models are given below: $\tilde{L}_{C P X}\left(M, x_{m}, f_{i}\right)$ where $M$ is either $d L_{g}, d L_{C O}$ or $d L_{D A E}$. Input features $M$ are time-averaged for each location $x_{m} . a_{i}$ are coefficients fit with the linear regression model and $N_{f}=13$ (amount of $1 / 3$ octave bands). Three cases are compared:

1. Single band fit: $\tilde{L}_{C P X}\left(M, x_{m}, f_{i}\right)=a_{0}+a_{i} M\left(x_{m}, f_{i}\right)$

2. Three bands fit: 


$$
\tilde{L}_{C P X}\left(M, x_{m}, f_{i}\right)= \begin{cases}a_{0}+a_{1} M\left(x_{m}, f_{1}\right)+a_{2} M\left(x_{m}, f_{2}\right), & i=1 \\ a_{0}+a_{i-1} M\left(x_{m}, f_{i-1}\right)+a_{i} M\left(x_{m}, f_{i}\right)+a_{i+1} M\left(x_{m}, f_{i+1}\right), & 1 \leq i \leq N_{f} \\ a_{0}+a_{N_{f}-1} M\left(x_{m}, f_{N_{f}-1}\right)+a_{N_{f}} M\left(x_{m}, f_{N_{f}}\right), & i=N_{f}\end{cases}
$$

3. CPX bands fit: $\tilde{L}_{C P X}\left(M, x_{m}, f_{i}\right)=a_{0}+a_{1} M\left(x_{m}, f_{1}\right)+\ldots+a_{N_{f}} M\left(x_{m}, f_{N_{f}}\right)$

The following procedure has been performed for 10 random splits of train and test segments:

1. Group segments by $1 \mathrm{~km}$ segments.

2. Within these $1 \mathrm{~km}$ segments, keep a consecutive $500 \mathrm{~m}$ segment. Throw out the rest of the segments from the CPX segments dataset.

3. Label big segments $80 \%$ train set and $20 \%$ test set.

4. Linear regression is performed on small $20 \mathrm{~m}$ segments

In addition, a set of CPX measurements on the same roads taken during 2017 was also available. A small offset due to the CPX measurement procedure was observed. Therefore, a single band linear regression model is also constructed to predict CPX 2019 from CPX 2017. This model can be interpreted as an expected change between consecutive CPX measurements and can be regarded as a frame of reference for the comparison between the opportunistic approach and CPX 2019.

In figure B.4 the accuracy of the CPX prediction is presented. The mean squared error (MSE) has been calculated for 10 test folds and mean and standard deviation are shown on the figure. Single band models are compared with single band models, the same goes for 3 band models and the all bands models. An unexpectedly large MSE (>2dB) is observed for modelling CPX 2019 based on measurements of CPX 2017. Several things could have happened: the roads have degraded, the road has been resurfaced, precise driving track was slightly different on worn roads, or calibration of the CPX device was different in 2017. The expected uncertainty on repeated CPX measurements itself is expected to be within $1 \mathrm{~dB}$ for overall levels [11. But at particular frequencies deviations e.g. between measurement devices may indeed be up to a few dB $[8]$.

For $f<1600 \mathrm{~Hz}$ the MSE of the single band model based on $d L_{D A E}$ is $1 \mathrm{~dB}$ smaller than the MSE for a model based on CPX 2 years earlier. However for higher frequencies, the MSE for the model based on CPX2017 is much smaller than the MSE of a prediction based on $d L_{D A E}$. It is expected that this is due to the differences between the standard CPX tire and the average tire in the opportunistic measurements. For most of the bands, the car offset model is superior to the speed correction: both in lower MSE value and in smaller spread. The additional improvement by the DEA model is less prominent. The MSE curves reach their lowest point at $1600 \mathrm{~Hz}$.

The all bands models are improvements over the 3 band model and the single band model (both in MSE and in variation between folds). This means that using multiple bands to predict one CPX $1 / 3$ octave band, improves results. The speed correction model and the car offset model give only slightly worse results than the DAE model, for the all bands case. This could indicate that the multiple linear regression model combining measurements at all frequency bands removes some of the measurement uncertainty at higher frequencies in a similar way as the DAE model. 


\section{Conclusions}

Today, CPX, SPB and CPB, are used as reliable methods to study the effect of road surface type on rolling noise production and to monitor road surface degradation. However, these methods are time consuming and expensive. Hence measurements are seldom repeated multiple times at one location and full coverage of all roads is out-of-reach. Hence we propose an opportunistic approach where cars that are on the road for different purposes are used for monitoring road surface state and its impact on rolling noise. In contrast to earlier attempts that tried to rely on smartphone sensors, a dedicated sound and vibration sensor box was designed and produced using consumer grade electronics and sensors. These sensor-boxes are placed in the trunk of passenger cars well shielded from wind and rain which allows them to be operated continuously and with little chance of mechanical damage. Future studies might transfer the setup to light and heavy trucks, since these vehicles produce a significant amount of rolling noise in main freight corridors.

Individual opportunistic measurements however introduce a lot of uncertainty due to uncontrolled measurement conditions: varying driving conditions (speed, acceleration, temperature, tire pressure...), confounders (engine noise and noise from within the car), the vehicle (engine, tire, transfer function to the trunk). Yet, the number of measurements obtained on a single stretch of road is often very high and thus data-driven calibration methods can be introduced to calibrate the measurements. In this paper, three calibration methods have been compared: a speed correction based on a Generalized Additive Model, an additional car offset model and a de-noising auto encoder (DAE). The DAE is a deep neural network which has to goal of transforming observations of one vehicle into a set of observations of the entire vehicle fleet at reference conditions (reference temperature...). It is by far the most advanced approach. On training, the DAE relies on a partial overlap between the observations by different vehicles at some locations.

Several methods for assessing the effectiveness of removing known dependencies, calibrating and de-noising are explored. Variability between measurement at the same location (i.e. an indication of measurement imprecision) has to be lowered while preserving the variability of measurements between locations (i.e. an indication of measurement sensitivity). The DAE reduces within-location variability to less than one $\mathrm{dB}$, however variability over locations is also reduced. Nevertheless, the ratio of both (i.e. sensitivity over imprecision) is significantly higher than for the other calibration methods. In addition, applying the DAE improves convergence with measurement error on the mean dropping below $0.25 \mathrm{~dB}$ even after a few observations, whereas getting measurement error below $1 \mathrm{~dB}$ would require 100 passages for the raw measurement. Therefore fewer measurements at the same location (car passages) are necessary to obtain the noise label. This also allows monitoring trends over time. This is illustrated by observing trends at locations where road construction works resulted in a new road surface. Analysing seasonal trends shows that the DAE removes seasonal variations from the measurements, probably based on temperature information from nearby meteorological stations that is included as a contextual variable in the DAE.

Finally, the obtained rolling noise factors for each $20 \mathrm{~m}$ road segment are validated against externally obtained close-proximity (CPX) data. Applying the DAE reduces the mean squared error (MSE) between the opportunistic data and CPX to a level below the MSE between CPX measurements conducted in 2017 and 2019 for frequencies 
below $1600 \mathrm{~Hz}$. Above $2000 \mathrm{~Hz}$ the proposed method deviates more from the CPX data. This might be attributed to the range of different tires that is used in our opportunistic approach in comparison to the single (or dual) tire assessment used by the CPX measurement.

The DAE is a data driven neural network approach which is expected to keep improving as more training data become available. Indeed, in the coming years, more data should become available, since the system is being rolled out into many more cars. In particular: the calibration and de-noising of edge cases (roads which do not occur a lot) will be improved.

\section{Acknowledgments}

This research received funding from the Research Foundation Flanders (FWO-Vlaanderen) [G0D5215N], ERC Runner-up project MAESTRO. In addition, the data has been provided by the ICON project MobiSense [HBC.2017.0155], supported by IMEC and Flanders Innovation Entrepreneurship (Vlaio). Finally, we thank Barbara Vanhooreweder and Ann Buytaert (Agentschap Wegen en Verkeer, Vlaanderen) for providing the CPX-data.

Declaration of interest: the authors declare a stock ownership in / employment by ASAsense cvba which is bringing the discussed measurement method on the market.

Van Hauwermeiren, Wout: Writing of Manuscript, Software; Botteldooren, Dick: Supervision, Conceptualization; Filipan, Karlo: Data Curation, Review of Manuscript; De Coensel, Bert: Supervision, Conceptualization, Funding acquisition 


\section{References}

[1] K. Heutschi, E. Bühlmann, J. Oertli, Options for reducing noise from roads and railway lines, Transportation Research Part A: Policy and Practice 94 (2016) 308 - 322.

[2] U. Sandberg, Consumer Label for Tyres in Europe, Tech. rep., Swedish National Road and Transport Research Institute (March 2008).

[3] H. Bendtsen, E. Kohler, Q. Lu, B. Rymer, California-denmark study on acoustic aging of road pavements, Transportation Research Record: Journal of the Transportation Research Board 2158 (2010) 122-128.

[4] S. Kephalopoulos, M. Paviotti, F. Anfosso-Lédée, Common noise assessment methods in europe (cnossos-eu) (2012).

[5] A. M. T. Bennert, D. Hanson, N. Vitillo, Influence of pavement surface type on tire/pavement generated noise, Journal of Testing and Evaluation 33 (2005) 94-100.

[6] M. Conter, R. Wehr, C. Aichinger, U. Sandberg, L. Goubert, P. Mioduszewski, R. Holck Skov, F. AnfossoLédée, P. Morgan, ROSANNE project: new procedure for noise characterization of road surfaces in Europe.

[7] F. Anfosso-Lédée, G. Dutilleux, M. Conter, Compatibility of the ROSANNE noise characterization procedure for road surfaces with CNOSSOS-EU model.

[8] T. Vieira, U. Sandberg, Close Proximity (CPX) Round Robin test: Comparison of results from four different CPX trailers measuring noise properties of 10 Swedish road surfaces, in: 48th International Congress and Exhibition on Noise Control Engineering (Inter.noise 48, INTER-NOISE and NOISE-CON Congress and Conference Proceedings, 2019.

[9] Acoustics - Measurement of the influence of road surfaces on traffic noise - Part 2: The close-proximity method, Standard, International Organization for Standardization, Geneva, CH (2017).

[10] Acoustics - Measurement of the influence of road surfaces on traffic noise - Part 3: Reference tyres, Standard, International Organization for Standardization, Geneva, CH (2017).

[11] E. Bühlmann, Improvements in the CPX method and its ability to predict traffic noise emissions, in: 48th International Congress and Exhibition on Noise Control Engineering (Inter.noise 48), INTER-NOISE and NOISE-CON Congress and Conference Proceedings, 2019.

[12] G. Licitra, L. Teti, M. Cerchiai, F. Bianco, The influence of tyres on the use of the CPX method for evaluating the effectiveness of a noise mitigation action based on low-noise road surfaces, Transportation Research Part D: Transport and Environment 55 (2017) $217-226$. 
[13] J. V. den Bossche, J. Theunis, B. Elen, J. Peters, D. Botteldooren, B. D. Baets, Opportunistic mobile air pollution monitoring: A case study with city wardens in Antwerp, Atmospheric Environment 141 (2016) 408 $-421$.

[21] W. Van Hauwermeiren, D. Botteldooren, K. Filipan, B. De Coensel, Opportunistic Method for Road Surface Noise Labelling: Data Cleaning, in: Proceedings of the 49th International Congress and Exposition on Noise Control Engineering, 2020.

[22] L. Balzano, R. Nowak, Blind calibration of sensor networks, Association for Computing Machinery, New York, NY, USA, 2007.

[23] J. Lipor, L. Balzano, Robust blind calibration via total least squares, in: 2014 IEEE International Conference on Acoustics, Speech and Signal Processing (ICASSP), 2014, pp. 4244-4248. 
[24] A. W. Alhashimi, Statistical sensor calibration algorithms, Ph.D. thesis, Luleå University of Technology, Luleå, Sweden (2018).

[25] Y. Wang, A. Yang, X. Chen, P. Wang, Y. Wang, H. Yang, A deep learning approach for blind drift calibration of sensor networks, IEEE Sensors Journal 17 (13) (2017) 4158-4171.

[26] J. Trogh, Location tracking in indoor and outdoor environments based on the viterbi principle, Ph.D. thesis, Ghent University (2019).

[27] G. Zambon, H. E. Roman, R. Benocci, Scaling model for a speed-dependent vehicle noise spectrum, Journal of Traffic and Transportation Engineering (English Edition) 4 (3) (2017) 230 - 239.

[28] F. Pedregosa, G. Varoquaux, A. Gramfort, V. Michel, B. Thirion, O. Grisel, M. Blondel, P. Prettenhofer, R. Weiss, V. Dubourg, J. Vanderplas, A. Passos, D. Cournapeau, M. Brucher, M. Perrot, E. Duchesnay, Scikit-learn: Machine learning in Python, Journal of Machine Learning Research 12 (2011) 2825-2830.

[29] X. Glorot, A. Bordes, Y. Bengio, Deep sparse rectifier neural networks, in: G. Gordon, D. Dunson, M. Dudík (Eds.), Proceedings of the Fourteenth International Conference on Artificial Intelligence and Statistics, Vol. 15 of Proceedings of Machine Learning Research, PMLR, Fort Lauderdale, FL, USA, 2011, pp. 315-323.

[30] I. Goodfellow, Y. Bengio, A. Courville, Deep Learning, MIT Press, 2016, http://www.deeplearningbook. org.

[31] N. Srivastava, G. Hinton, A. Krizhevsky, I. Sutskever, R. Salakhutdinov, Dropout: A simple way to prevent neural networks from overfitting, J. Mach. Learn. Res. 15 (1) (2014) 1929-1958.

[32] D. P. Kingma, J. Ba, Adam: A Method for Stochastic Optimization, arXiv e-prints (Dec. 2014).

[33] D. Arpit, S. Jastrzebski, N. Ballas, D. Krueger, E. Bengio, M. S. Kanwal, T. Maharaj, A. Fischer, A. Courville, Y. Bengio, S. Lacoste-Julien, A closer look at memorization in deep networks, in: D. Precup, Y. W. Teh (Eds.), Proceedings of the 34th International Conference on Machine Learning, Vol. 70 of Proceedings of Machine Learning Research, PMLR, International Convention Centre, Sydney, Australia, 2017, pp. 233-242.

[34] X. Glorot, Y. Bengio, Understanding the difficulty of training deep feedforward neural networks, in: Y. W. Teh, M. Titterington (Eds.), Proceedings of the Thirteenth International Conference on Artificial Intelligence and Statistics, Vol. 9 of Proceedings of Machine Learning Research, PMLR, Chia Laguna Resort, Sardinia, Italy, 2010, pp. 249-256.

[35] F. Anfosso-Lédée, Y. Pichaud, Temperature effect on tyre-road noise, Applied Acoustics 68 (1) (2007) 1 - 16.

[36] R. Wehr, A. Fuchs, C. Aichinger, A combined approach for correcting tyre hardness and temperature influence on tyre/road noise, Applied Acoustics 134 (2018) 110 - 118. 
[37] J. Alonso, J. López, I. Pavón, M. Recuero, C. Asensio, G. Arcas, A. Bravo, On-board wet road surface identification using tyre/road noise and support vector machines, Applied Acoustics 76 (2014) 407-415.

\section{Appendix A. Code}

Listing A.1: Training algorithm of the De-noising Auto Encoder (pseudo-Python code)

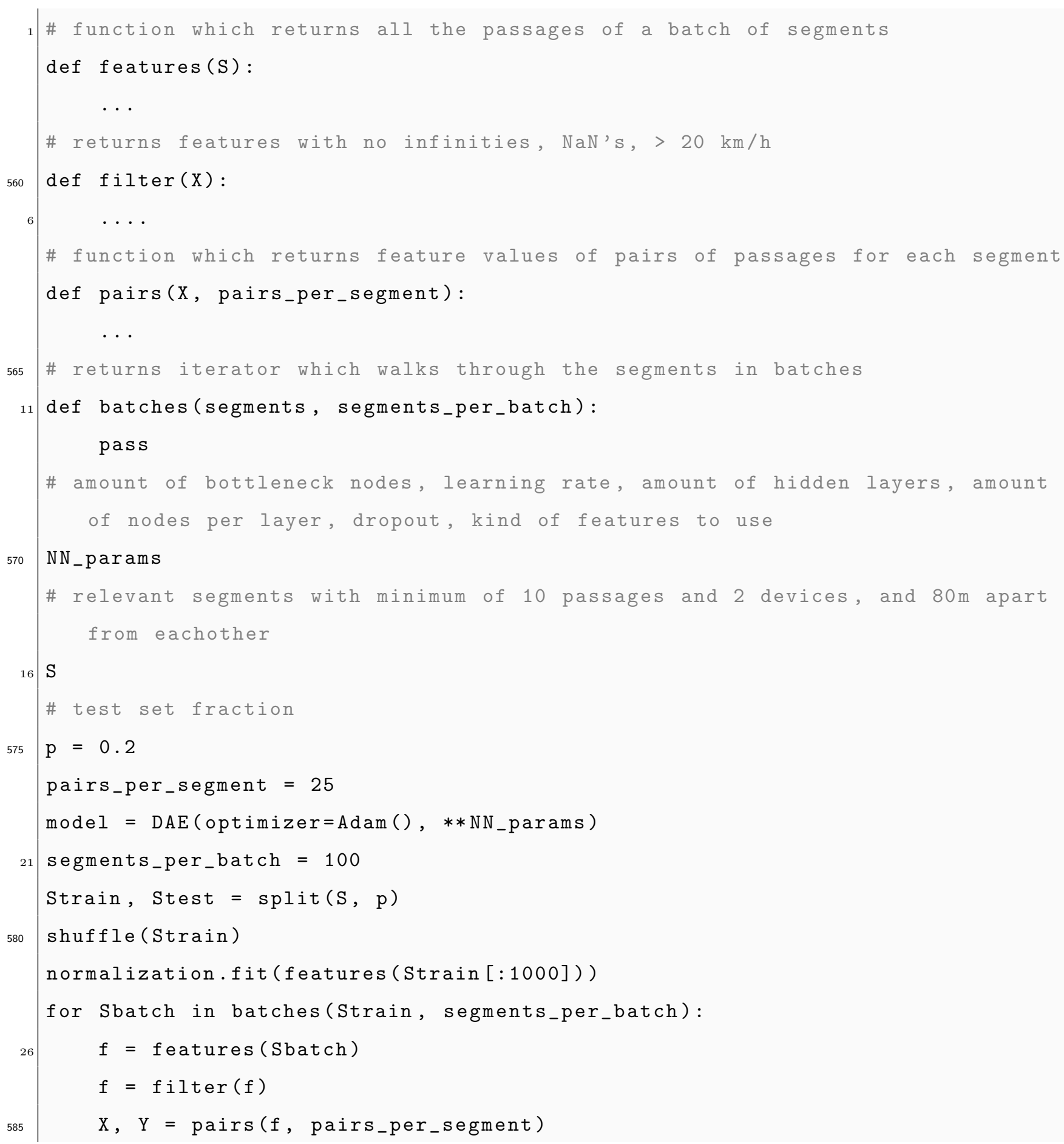




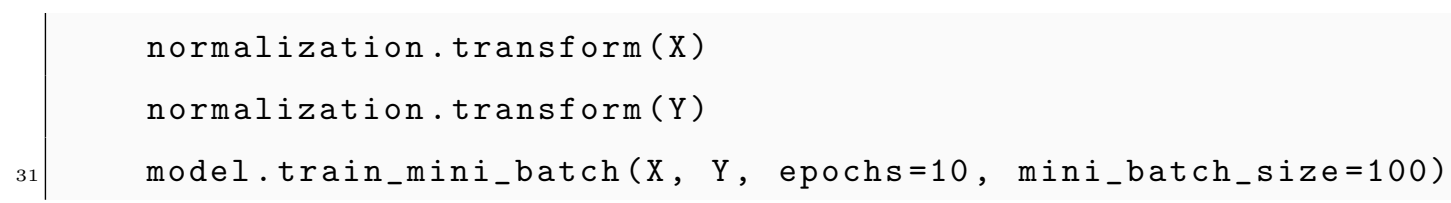

\section{Appendix B. Figures}
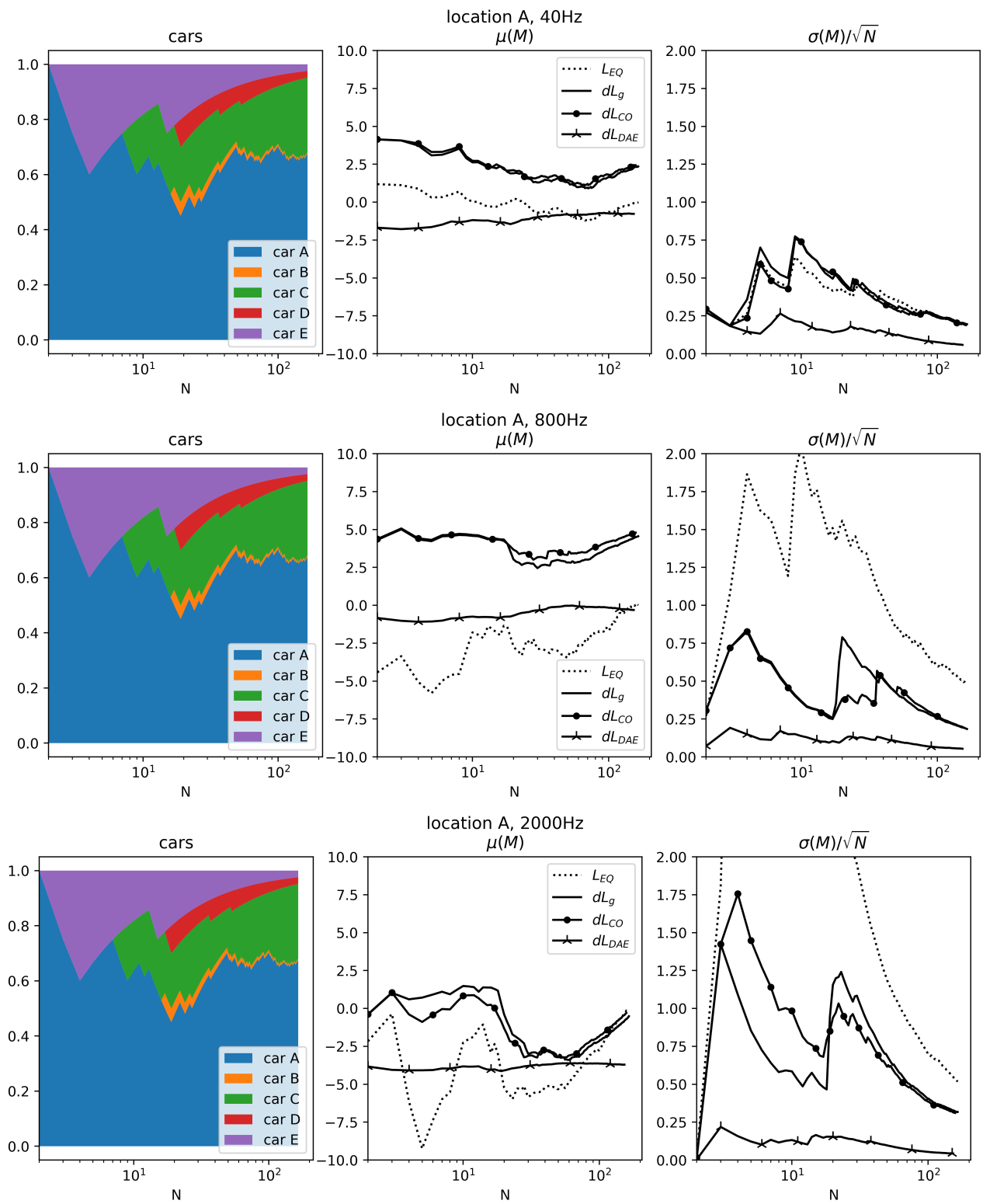

Figure B.1: $N$ is the amount of passages taken (logarithmic scale). Left: fraction of observations measured by each sensing system, middle: mean value, right: standard error. Average value of $L_{E Q}$ has been subtracted from $L_{E Q}$ to be able to plot $L_{E Q}$ on the same

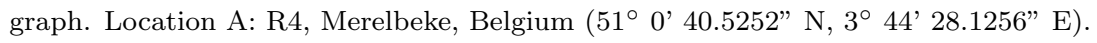



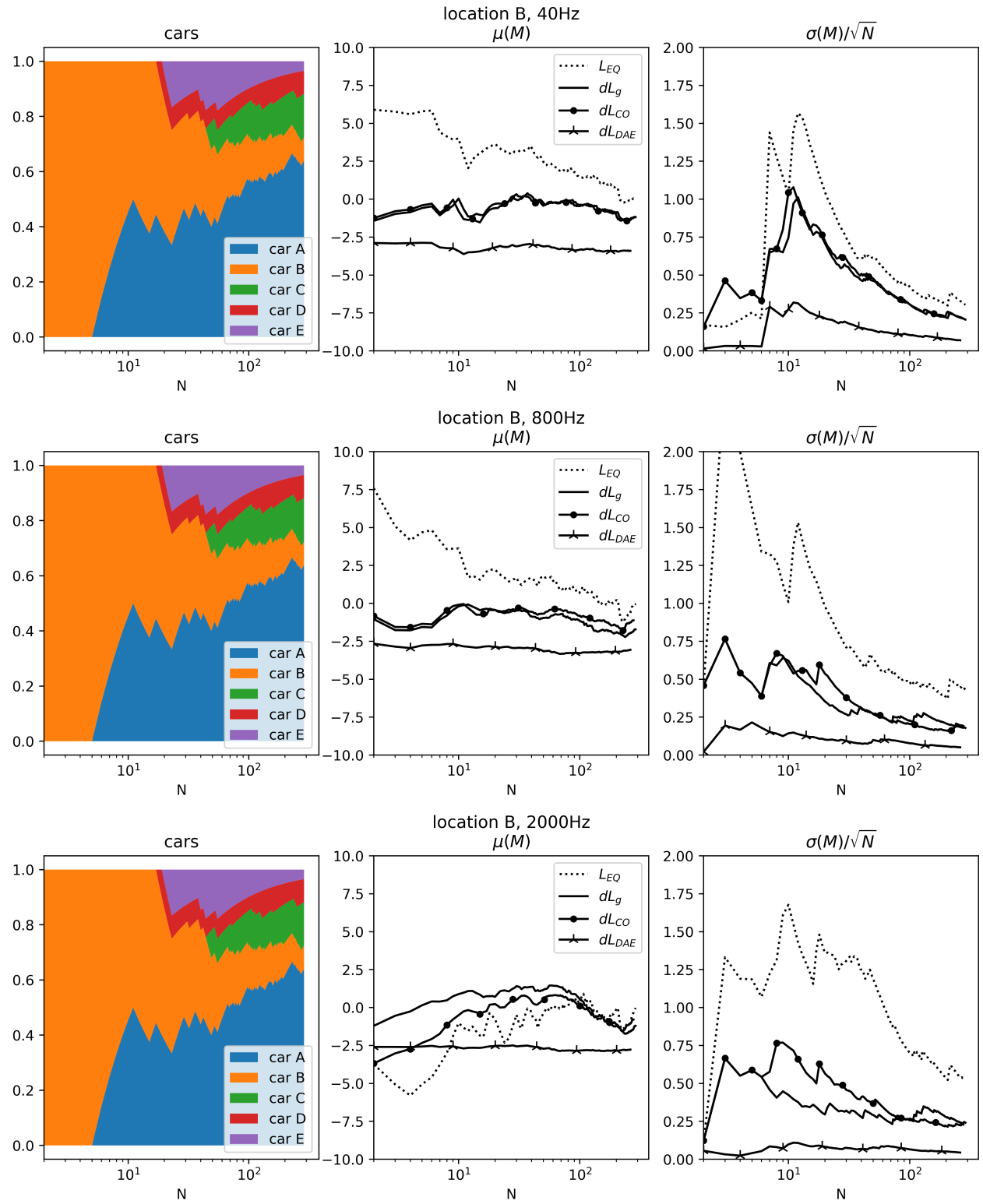

Figure B.2: $N$ is the amount of passages taken (logarithmic scale). Left: fraction of observations measured by each sensing system, middle: mean value, right: standard error of sample. Average value of $L_{E Q}$ has been subtracted from $L_{E Q}$ to be able to plot $L_{E Q}$ on the same graph. Location B: B401, exit to E17, Gentbrugge, Belgium (51ㄷ 1' 12.0894" N, 3 43' 55.0986” E). 


\section{Car a, $800 \mathrm{~Hz}$}
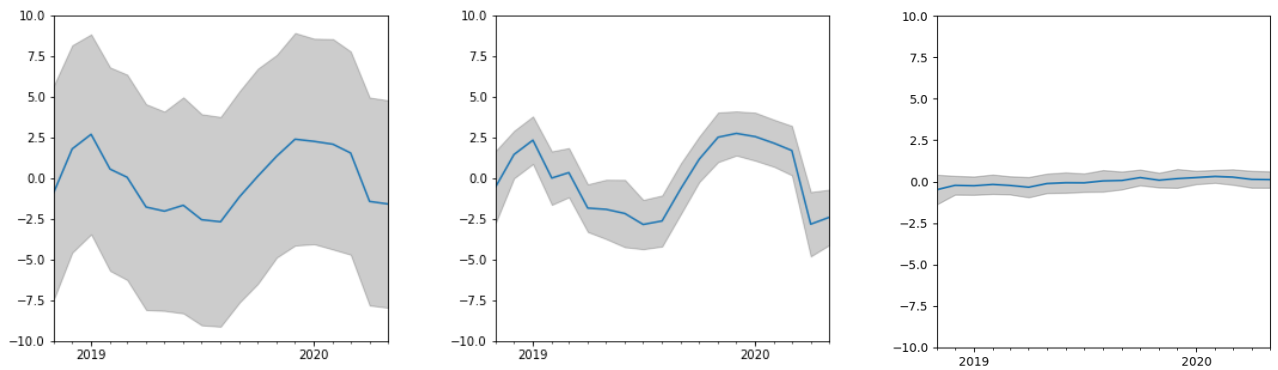

Car c, $800 \mathrm{~Hz}$
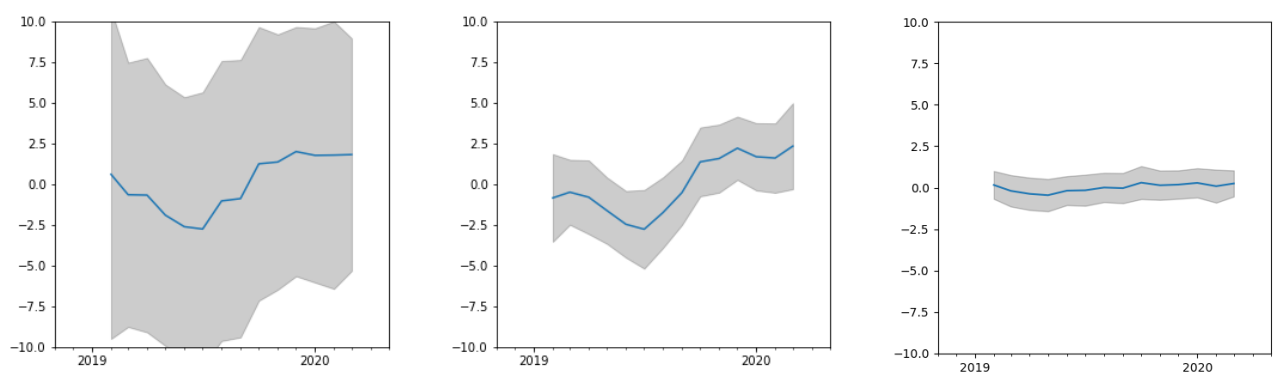

(a) $L_{E Q}\left(x_{m}\right)-\mu\left(L_{E Q}\left(x_{m}\right)\right)$

(b) $d L_{g}\left(x_{m}\right)$

(c) $\left.d L_{D A E}\left(x_{m}\right)\right)$

Car a, $2000 \mathrm{~Hz}$
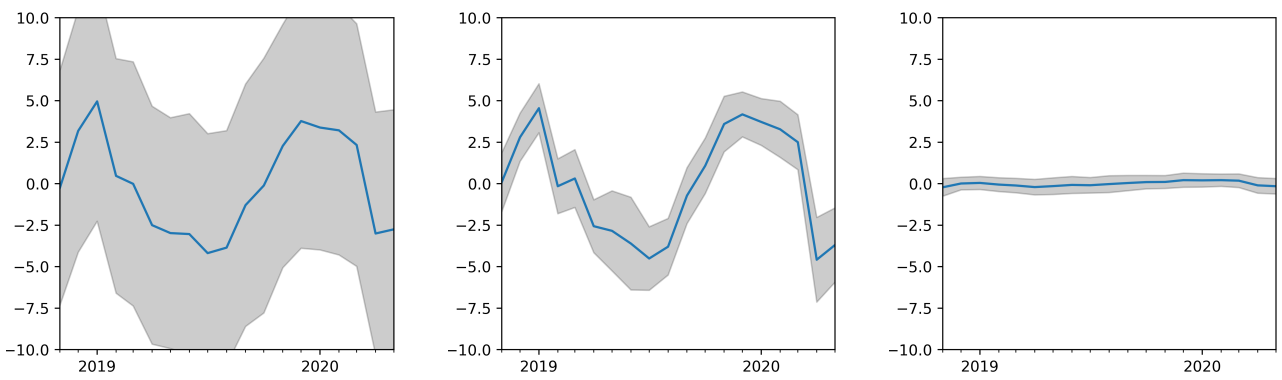

Car c, $2000 \mathrm{~Hz}$
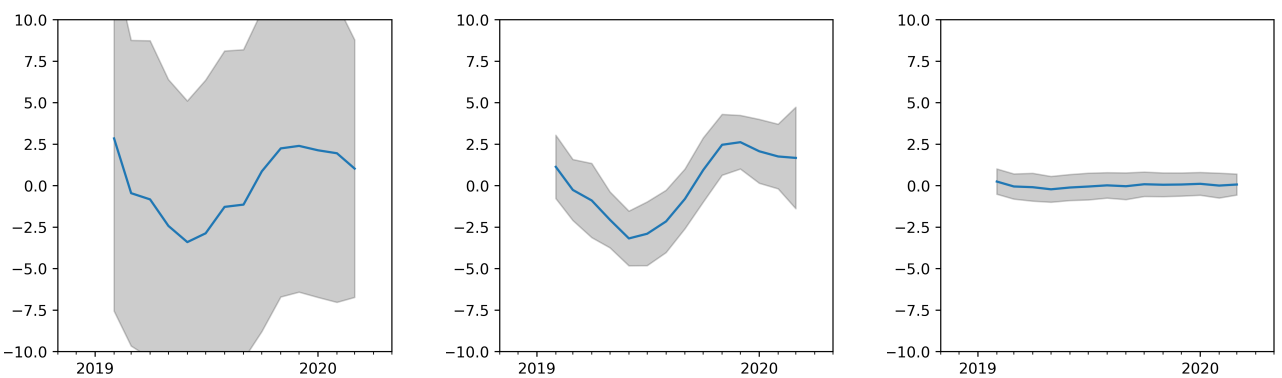
(d) $L_{E Q}\left(x_{m}\right)-\mu\left(L_{E Q}\left(x_{m}\right)\right)$
(e) $d L_{g}\left(x_{m}\right)$
(f) $d L_{D A E}\left(x_{m}\right)$

Figure B.3: Seasonal variations observed by device a and device c at $800 \mathrm{~Hz}$ and $2000 \mathrm{~Hz}$. Locations $x_{m}$ with more than 100 passages made by the selected device are included. Then, monthly averages (blue line) are computed and plotted. $\pm o(M)$ is indicated as a gray zone around the monthly average. 


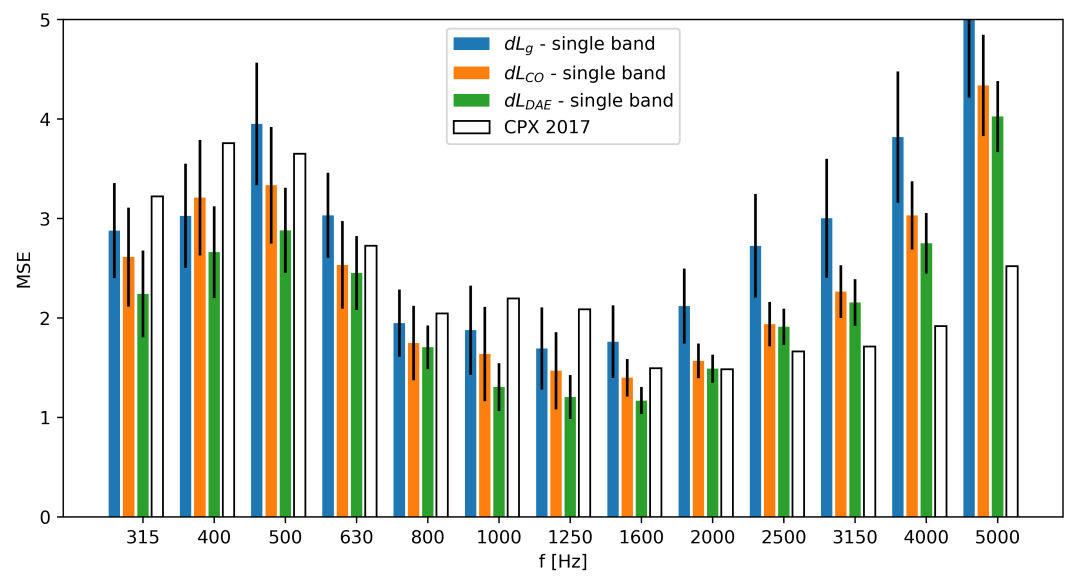

(a) single band

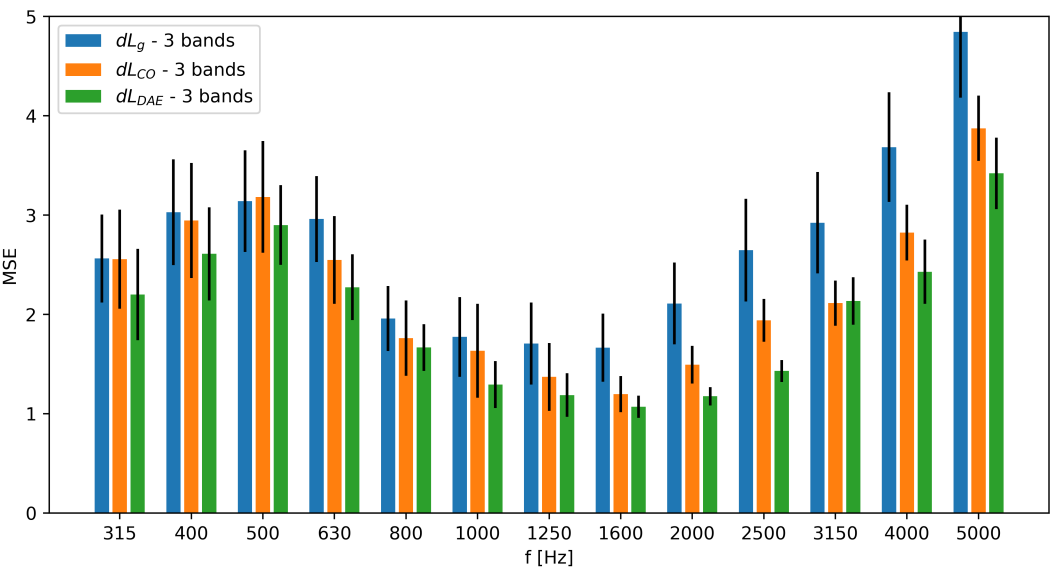

(b) 3 bands

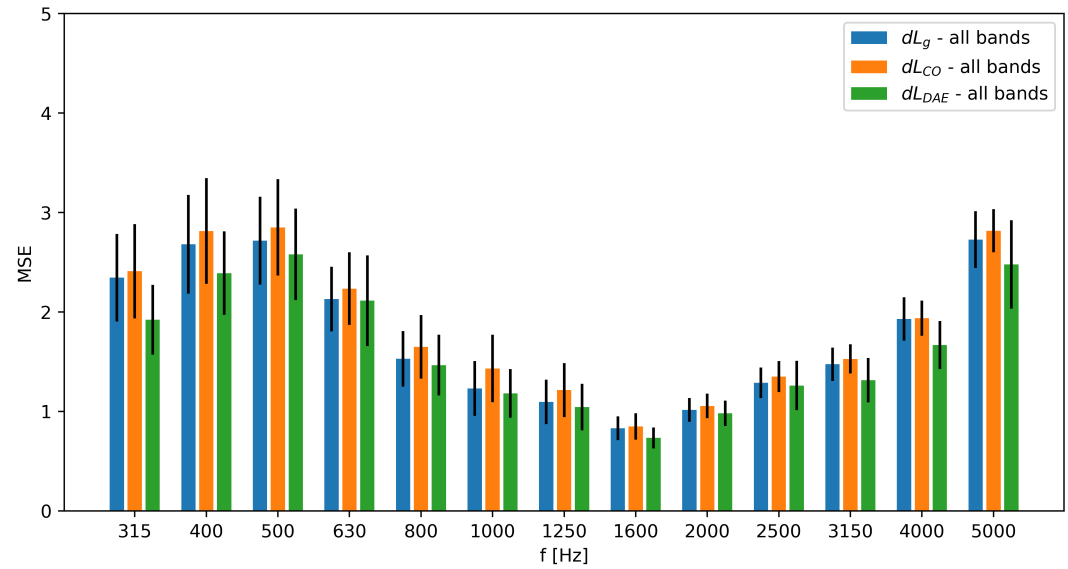

(c) all bands

Figure B.4: Average over validation (test set) mean squared error (MSE) obtained from 10 randomized train test set splits of the CPX dataset. The black bars at the top of each bar represents $\pm \sigma(M S E)$, the standard deviation of the validation MSE over different folds. 